\title{
Extended Weyl-Heisenberg algebra, phase operator, unitary depolarizers and generalized Bell states
}

\author{
M. Daoud ${ }^{1 *}$ and E.H. El Kinani ${ }^{2}$ \\ 1 Max Planck Institute for Physics of Complex Systems, Nöthnitzer Str. 38, \\ D-01187 Dresden, Germany \\ 2 Moulay Ismail University, Faculty of Sciences and Technics, PO Box 509, \\ Boutalamine, Errachidia, Morocco
}

\begin{abstract}
Finite dimensional representations of extended Weyl-Heisenberg algebra are studied both from mathematical and applied viewpoints. They are used to define unitary phase operator and the corresponding eigenstates (phase states). It is also shown that the unitary depolarizers can be constructed in a general setting in terms of phase operators. Generation of generalized Bell states using the phase operator is presented and their expressions in terms of the elements of mutually unbiased bases are given.
\end{abstract}

\footnotetext{
${ }^{*}$ Faculté des Sciences, Département de Physique, Agadir, Morocco; email: daoud@pks.mpg.de
} 


\section{Introduction}

The existence of phase operator, for quantized single mode electromagnetic field, was first postulated by Dirac [1]. However, since the harmonic oscillator spectrum is infinite, the creation and annihilation operators do not admit a naive polar decomposition. The infinite dimensional character of the harmonic oscillator Fock space constitutes a drawback in defining a phase operator in a consistent way [2, 3, 4]. To overcome this problem, Pegg and Barnett considered a truncated oscillator of finite dimension and defined the Hermitian phase operator [5]. But the truncated oscillator algebra has also one disadvantage from the algebraic point of view: its operators do not form a closed algebra. Moreover, the truncated oscillator is not the only way to realize the Pegg-Barnett phase operator. Indeed, many approaches were proposed in the literature as for instance the refined version of phase operator proposed in [6]. This approach is based on the generalized Weyl-Heisenberg algebra which provides finite dimensional representations and where the hermiticity of the phase operator is automatically ensured.

In Section 1, we consider a particular, but nevertheless very large class of extended Weyl-Heisenberg algebra. It comprises many well-known oscillator algebras, as particular or limiting cases, constructed in [6, 7, 8, 9, 10, 11. We shall pay attention to extended Weyl-Heisenberg algebras possessing finite dimensional representations. Based on polar decomposition of raising and lowering generators, we construct algebraically the Hermitian phase operator. We discuss the temporal stability of the phase states (the eigenstates of the phase operator) and particularly the key role of the phase parameter ensuring this temporal stability. We study some quantum properties of the phase operator and compare with the results obtained using the Pegg-Barnett formalism.

Section 2 of this work deals with the connection between the unitary phase operators and the so-called unitary depolarizer operators. This is mainly motivated by the fact that for finite dimensional systems, unitary operations have a prominent importance in quantum information processing. In particular, unitary depolarizer operators are useful for quantum information processing as for instance quantum teleportation, quantum dense coding [12, 13, 14] as well as in investigating quantum optical systems. Note also that the notion of unitary depolarizer operators is deeply related to the entanglement phenomenon which has attracted a considerable attention as a crucial resource for quantum information processing involving higher dimensional systems. Here, we show clearly that unitary depolarizer operators can be constructed from the phase operator using the algebraic structures of the extended Weyl-Heisenberg algebra. We stress that our construction generalizes one presented recently in [15].

In Section 3, we show that the generalized Bell states introduced in [16] can be generated by means the generalized phase operator. It is important to mention that many efforts have been devoted, during the last years, to such states to understand bipartite entanglement in higher dimensional systems [17, 18, 19, 20, 21]. The generalized Bell states provide a basis of maximally entangled states in a finite dimensional Hilbert space. In this respect, we examine interesting entanglement properties of some particular superpositions of generalized Bell states. Finally, since mutually unbiased bases [22, 23] can be derived from the phase states [6] and the generalized Bell states are defined by means of phase operator, it is natural to ask about the connection between generalized Bell states and vectors of mutually unbiased bases. In this sense, inspired by the results recently presented in [24], we 
derive such a relation taking advantage of the relation, between phase states and mutually unbiased bases, derived in [6]. This establishes a useful link between mutually unbiased vectors containing single particle information and Bell vectors reflecting the maximal bipartite entanglement. Concluding remarks close this Letter.

\section{Extended Weyl-Heisenbeg algebra and phase states}

In this section we first introduce the extended Weyl-Heisenberg algebra. We indicate the constraints of the structure relations to get finite dimensional representations suitable to ensure the unitarity of the phase operator. Then, we construct the temporally stable phase states. The expectation value of the Hermitian phase operator is explicitly derived.

\subsection{Finite Fock space for extended Weyl-Heisenberg algebra}

The extended Weyl-Heisenberg algebra is an associative algebra over the complex field $\mathbb{C}$, with generators $\left\{a^{+}, a^{-}, N\right\}$ and the unit $\mathbf{I}$, satisfying the structure relations

$$
\left[N, a^{-}\right]=-a^{-}, \quad\left[N, a^{+}\right]=+a^{+}, \quad\left[a^{-}, a^{+}\right]=G(N)
$$

where the non-negative function $G(N)=(G(N))^{\dagger}$ is some Hermitian analytic function of the number operator $N$. It is clear that for $G(N)=\mathbf{I}$, one recovers the usual harmonic oscillator algebra. Also, this algebra reproduces the $W_{k}$ algebra discussed in [11] in the context of fractional supersymmetric quantum mechanics of order $k$ and covers the extended harmonic oscillator worked out in [8, 9,

Let us consider the abstract Fock representation of this algebra by means of a complete set of orthonormal states $\{|n\rangle, n \in \mathbb{N}\}$ which are eigenstates of the number operator $N, N|n\rangle=n|n\rangle$. In this representation, the vacuum state is defined as $a^{-}|0\rangle=0$ and the orthonormalized eigenstates are constructed by successive applications of the creation operator $a^{+}$. Indeed, we define the actions of creation and annihilation operators as

$$
a^{-}|n\rangle=\sqrt{F(n)} e^{i[F(n)-F(n-1)] \varphi}|n-1\rangle, \quad a^{+}|n\rangle=\sqrt{F(n+1)} e^{-i[F(n+1)-F(n)] \varphi}|n+1\rangle
$$

where the structure function $F($.$) is an analytic function and should satisfy the conditions$

$$
F(0)=0 \quad \text { and } \quad F(n)>0, \quad n=1, \ldots
$$

In what follows we shall denote the Fock space as $\mathcal{F}$. On this space the operators $a^{+}$and $a^{-}$are mutually adjoint, $a^{+}=\left(a^{-}\right)^{\dagger}$. It is easy to check that the structure function $F($.$) satisfies the$ following recursion formula

$$
F(n+1)-F(n)=G(n),
$$

which gives by simple iteration

$$
F(n)=\sum_{m=0}^{n-1} G(m) .
$$


We restrict ourselves here to generalized oscillator algebra defined through structure functions obeying the condition

$$
F(2 s+1)=0
$$

where $2 s$ is a positive integer. We assume that $F(n)$ does not have zeros at non-negative integers values of $n$, except 0 and $2 s+1$. It follows that, in this case, the creation-annihilation operators satisfy the nilpotency relations $\left(a^{-}\right)^{2 s+1}=\left(a^{+}\right)^{2 s+1}=0$. This means that the corresponding representation is $(2 s+1)$-dimensional. It is interesting to note that using (5), the condition (6) can be written as

$$
\operatorname{Tr} G=0
$$

where the trace is over the $(2 s+1)$-dimensional Fock space. The structure function $F(N)$ can be factorized as

$$
F(N)=\frac{N}{2 s}(2 s+1-N) f(N)
$$

where $f(N)$ does not have vanishing eigenvalues for $0 \leq n \leq 2 s$. Obviously, this algebra covers the usual harmonic oscillator for $f(N)=\mathbf{I}$ when $s$ is large. It also includes the finite dimensional oscillator algebra $\mathcal{A}_{\kappa}(\kappa<0)$ defined in [6] when $f(N)=\mathbf{I}$ and $\kappa=-1 / 2 s$. Of course, as evoked above, the algebra introduced here covers many other variants of generalized harmonic oscillators, but we quote only the ones possessing finite dimensional representations which are interesting in the context of quantum optics.

The algebra (1) defined by means of the structure function $F(N)$ of the form (8) can be realized in terms of the usual single photon operator. For that end, one can write the creation and annihilation operators as

$$
a^{-}=b^{-} \sqrt{1-\frac{N-1}{2 s}} \sqrt{f(N)} \quad a^{+}=\sqrt{f(N)} \sqrt{1-\frac{N-1}{2 s}} b^{+}
$$

where $b^{-}$and $b^{+}$and the number operator $N=b^{+} b^{-}$are the usual harmonic oscillator operators. As example of the function $f(N)$, one may choose the function

$$
f(N)=\left(b^{-}\right)^{m}\left(b^{+}\right)^{m} \quad m \in \mathbb{N}
$$

introduced in [7] to construct the multiphoton realization of generalized oscillator algebra. For our purpose the explicit form of the function $f(N)$ is unessential through this work (except in the last subsection of the section 3 where we set $f(N)=\mathbf{I})$.

Using the algebraic structure of the generalized oscillator algebra, one can introduce an operator which generalizes the Hamiltonian $a^{+} a^{-}$for the one-dimensional harmonic oscillator. Starting from

$$
a^{+} a^{-}|n\rangle=F(n)|n\rangle \Longrightarrow H(N) \equiv F(N)=a^{+} a^{-}
$$

We refer $H(N)$ to as an Hamiltonian associated with the extended Weyl-Heisenberg algebra. The eigenvalue equation

$$
H(N)|n\rangle=F(n)|n\rangle=\frac{n}{2 s}(2 s+1-n) f(n)|n\rangle
$$


gives the energies of a quantum dynamical system described by the Hamiltonian operator $H(N)$. It is obvious that for $f(N)=\mathbf{I}$ and $s \longrightarrow \infty$, the Hamiltonian reads

$$
H(N)=\sum_{n=0}^{\infty} n|n\rangle\langle n|,
$$

and for $f(N)=\mathbf{I}$ and $s$ finite, one has

$$
H(N)=\sum_{n=0}^{2 s} \frac{n}{2 s}(2 s+1-n)|n\rangle\langle n|,
$$

which coincides with the Hamiltonian introduced in [6]. In this particular case, it is interesting to stress that the ladder operators $a^{+}$and $a^{-}$can be related to Stokes operators introduced in [25, 26] to define a unitary operator representing the exponential of the phase difference between two modes of the electromagnetic field. This relation may be expressed as

$$
s_{+}=\sqrt{s} a_{+} \quad s_{-}=\sqrt{s} a_{-} \quad s_{3}=\frac{1}{2}(N-s)
$$

and one can simply verify that the Stokes generators $s_{+}, s_{-} s_{3}$ close the $s u(2)$ algebra. It follows that, in the special case where $f(N)=N(2 s+1-N) / s$, the extended Weyl-Heisenberg generators can be realized à la Holstein-Primakoff by means of two independent ordinary bosons (two electromagnetic field modes). Remark that the parameter $s$ can be viewed as controlling the deformation scheme. Indeed, when $s$ becomes large one has the standard harmonic oscillator algebra. We mention that the idea of extended Weyl-Heisenberg is mainly inspired by the polynomial deformation of Lie algebras introduced in [27, 28] and extensively discussed in the context of quantum algebras [29, 30, 31]. Finally, we emphasize that quantum systems described by a nonlinear Hamiltonian $H(N)$ are familiar in the context of nonlinear quantum optics as for instance electromagnetic field propagating trough a nonlinear Kerr medium 32 .

\section{$2.2 \quad$ Temporally stable phase states}

The Hilbert space $\mathcal{F}$ is $(2 s+1)$-dimensional. The actions of $a^{-}$and $a^{+}$on $\mathcal{F}$ are given by the equation (2) supplemented by

$$
a^{+}|2 s\rangle=0
$$

which easily follows from the calculation of $\left\langle 2 s\left|a^{-} a^{+}\right| 2 s\right\rangle$.

Let us consider the polar decomposition of the creation $a^{+}$and annihilation $a^{-}$operators:

$$
a^{-}=E \sqrt{F(N)} \Leftrightarrow a^{+}=\sqrt{F(N)}(E)^{\dagger} .
$$

It follows that the operator $E$ satisfies

$$
E|n\rangle=e^{i[F(n)-F(n-1)] \varphi}|n-1\rangle
$$

for $n=1,2, \ldots, 2 s$. For $n=0$, the action of $E$ is defined by

$$
E|0\rangle=e^{i[F(0)-F(2 s)] \varphi}|2 s\rangle
$$


so that (16) is valid modulo $2 s+1$. It follows that we have

$$
(E)^{\dagger}|n\rangle=e^{-i[F(n+1)-F(n)] \varphi}|n+1\rangle,
$$

where $n+1$ should be understood modulo $2 s+1$. It is important to stress that the operator $E$ is unitary. Therefore, equation (15) constitutes a polar decomposition of creation and annihilation operators $a^{+}$and $a^{-}$.

To construct the phase states associated with the finite dimensional algebra under consideration, let us look to the eigenstates of the operator $E$. For this, one has to solve the eigenvalue equation

$$
E|z\rangle=z|z\rangle, \quad|z\rangle=\sum_{n=0}^{2 s} C_{n} z^{n}|n\rangle
$$

with $z \in \mathbb{C}$. According to the method developed in [6], it is easy to see that the eigenvalues $z$ should satisfy the discretization condition

$$
z^{2 s+1}=1
$$

and subsequently the complex variable $z$ is a root of unity given by

$$
z=q^{m} \quad m=0,1, \ldots, 2 s,
$$

where

$$
q:=e^{2 \pi i /(2 s+1)}
$$

Then, the normalized eigenstates $|z\rangle \equiv|m, \varphi\rangle$ of $E$ are given by

$$
|m, \varphi\rangle=\frac{1}{\sqrt{2 s+1}} \sum_{n=0}^{2 s} e^{-i F(n) \varphi} q^{m n}|n\rangle .
$$

The states $|m, \varphi\rangle$, labeled by the parameters $m \in \mathbb{Z} /(2 s+1) \mathbb{Z}$ and $\varphi \in \mathbb{R}$, satisfy

$$
E|m, \varphi\rangle=e^{i \theta_{m}}|m, \varphi\rangle \quad \theta_{m}=m \frac{2 \pi}{2 s+1},
$$

which reflects that $E$ is indeed a phase operator. In the particular case $\varphi=0$, the states $|m, 0\rangle$ correspond to an ordinary discrete Fourier transform of the basis $\{|n\rangle: n=0,1, \ldots, 2 s\}$ of the $(2 s+1)$-dimensional space $\mathcal{F}$. At this level, we would like to emphasize the key role of the parameter $\varphi$ introduced from the beginning in the definition of actions of creation and annihilation operators (2). First, it ensures the temporal stability of the phase states $|m, \varphi\rangle$ under "time evolution":

$$
e^{-i H(N) t}|m, \varphi\rangle=|m, \varphi+t\rangle
$$

for any value of the real parameter $t$. Secondly, if one ignores the parameter $\varphi$,i.e. $\varphi=0$, the phase states (23) reduce to ones derived by Pegg and Barnett using the truncated harmonic oscillator [5]. This means that for two different extended Weyl-Heisenberg algebras characterized by different structure functions, we will end up with the same phase states. Then to differentiate between them, the phase parameter is necessary. In the same spirit, it must be noticed that the $S U(2)$ phase states 
obtained in 33] are similar to ones obtained by Pegg and Barnett despite the fact that the involved symmetries are different. Hence, to avoid such a problem, we introduced the parameter $\varphi$ which allows us to keep the trace of the symmetry and the dynamics of the system under consideration. Note also that the parameter $\varphi$ plays a key role in relating phase states and mutually unbiased bases [6]. The phase states have the following remarkable properties. For fixed $\varphi$, they satisfy the equiprobability relation

$$
|\langle n \mid m, \varphi\rangle|=\frac{1}{\sqrt{2 s+1}} \quad n, m \in \mathbb{Z} /(2 s+1) \mathbb{Z} .
$$

They constitute an orthonormal set, for fixed $\varphi$,

$$
\left\langle m, \varphi \mid m^{\prime}, \varphi\right\rangle=\delta_{m, m^{\prime}} \quad m, m^{\prime} \in \mathbb{Z} /(2 s+1) \mathbb{Z}
$$

and satisfy the closure property

$$
\sum_{m=0}^{2 s}|m, \varphi\rangle\langle m, \varphi|=I
$$

Finally, the overlapping between two phase states $\left|m^{\prime}, \varphi^{\prime}\right\rangle$ and $|m, \varphi\rangle$ is given by

$$
\left\langle m, \varphi \mid m^{\prime}, \varphi^{\prime}\right\rangle=\frac{1}{2 s+1} \sum_{n=0}^{2 s} q^{\rho\left(m-m^{\prime}, \varphi-\varphi^{\prime}, n\right)},
$$

where

$$
\rho\left(m-m^{\prime}, \varphi-\varphi^{\prime}, n\right)=-\left(m-m^{\prime}\right) n+\frac{2 s+1}{2 \pi}\left(\varphi-\varphi^{\prime}\right) F(n)
$$

and $q$ is defined in (22). This shows that the temporally stable phase states are not all orthogonal.

\subsection{Expectation value of the phase operator}

In the previous subsection, we defined the unitary phase operator and the phase states. We shall calculate the expectation value of the Hermitian phase operator $\Theta$ defined by

$$
E=e^{i \Theta}
$$

It is clear that the Hermitian phase operator can be written as

$$
\Theta=\sum_{m=0}^{2 s} \theta_{m}|m, \varphi\rangle\langle m, \varphi|
$$

where the summation is modulo $2 s+1$. Using the expression of the phase states (23), one obtains the expansion of the Hermitian phase operator, in the Fock states basis, as

$$
\Theta=\frac{2 \pi}{(2 s+1)^{2}} \sum_{n=0}^{2 s} \sum_{n^{\prime}=0}^{2 s} e^{-i\left[F(n)-F\left(n^{\prime}\right)\right] \varphi} \theta\left(n, n^{\prime}\right)|n\rangle\left\langle n^{\prime}\right|
$$

where the objects $\theta\left(n, n^{\prime}\right)$ are given by

$$
\theta\left(n, n^{\prime}\right)=\sum_{m=1}^{2 s+1} m q^{m\left(n-n^{\prime}\right)}=(2 s+1)\left[s \delta_{n, n^{\prime}}+\frac{q^{n-n^{\prime}}}{1-q^{n-n^{\prime}}}\left(1-\delta_{n, n^{\prime}}\right)\right] .
$$


Using this result, we are now in position to compute the phase-number commutator. In the number basis, it can be expressed as

$$
[\Theta, N]=\frac{2 \pi}{2 s+1} \sum_{n \neq n^{\prime}}^{2 s}\left[\frac{\left(n-n^{\prime}\right) q^{n-n^{\prime}}}{q^{n-n^{\prime}}-1}\right] e^{-i\left[F(n)-F\left(n^{\prime}\right)\right] \varphi}|n\rangle\left\langle n^{\prime}\right| .
$$

This equation shows that the diagonal matrix elements of the phase-number commutator vanish and coincide with the result obtained by Pegg and Barnett [5] when $\varphi=0$. This indicates clearly that the generalized Weyl-Heisenberg algebra gives an alternative way to deal with finite dimensional harmonic oscillator in investigating the phase properties of an electromagnetic field. In this respect, the form of the phase states suggests us to consider a normalized pure state of the form

$$
|\Phi\rangle=\sum_{n=0}^{2 s} \Phi_{n} e^{i n \alpha} e^{-i F(n) \varphi}|n\rangle
$$

to evaluate the phase probability distribution and the phase expectation value. The parameter $\alpha$ is real and the coefficients $\Phi_{n}$ are real and positive satisfying

$$
\sum_{n=0}^{2 s} \Phi_{n}^{2}=1
$$

The vector $|\Phi\rangle$ is similar to the so-called partial phase state introduced in [5] (up to phase factor involving $\varphi$ ) and the phase probability distribution is

$$
|\langle m, \varphi \mid \Phi\rangle|^{2}=\frac{1}{2 s+1}+\frac{2}{2 s+1} \sum_{n>n^{\prime}} \Phi_{n} \Phi_{n^{\prime}} \cos \left[\left(n-n^{\prime}\right)\left(\alpha-\theta_{m}\right)\right] .
$$

It is bounded as follows

$$
\frac{1}{2 s+1}\left[2-\left(\sum_{n=0}^{2 s} \Phi_{n}\right)^{2}\right] \leq|\langle m, \varphi \mid \Phi\rangle|^{2} \leq \frac{1}{2 s+1}\left(\sum_{n=0}^{2 s} \Phi_{n}\right)^{2} .
$$

Using (33), the expectation value of the Hermitian phase operator $\Theta$ reads

$$
\langle\Theta\rangle=\langle\Phi|\Theta| \Phi\rangle=\frac{2 \pi}{(2 s+1)^{2}} \sum_{n=0}^{2 s} \sum_{n^{\prime}=0}^{2 s} \Phi_{n} \Phi_{n^{\prime}} e^{i \alpha\left(n-n^{\prime}\right)} \theta\left(n, n^{\prime}\right)
$$

which can be written as a sum of two terms

$$
\langle\Theta\rangle=\langle\Theta\rangle_{\mathrm{d}}+\langle\Theta\rangle_{\mathrm{nd}}
$$

where $\langle\Theta\rangle_{\mathrm{d}}$ stands for diagonal contributions $\left(n=n^{\prime}\right)$ and $\langle\Theta\rangle_{\text {nd }}$ is the contribution from the offdiagonal terms. These quantities are given by

$$
\langle\Theta\rangle_{\mathrm{d}}=\frac{2 \pi s}{2 s+1}
$$

and

$$
\langle\Theta\rangle_{\mathrm{nd}}=\frac{2 \pi}{2 s+1} \sum_{n>n^{\prime}} \Phi_{n} \Phi_{n^{\prime}} \frac{\sin \left(\left(\alpha-\frac{\pi}{2 s+1}\right)\left(n-n^{\prime}\right)\right)}{\sin \left(\frac{\pi}{2 s+1}\left(n-n^{\prime}\right)\right)} .
$$

It is clear that for $\alpha=\pi /(2 s+1)$, the off-diagonal contribution vanishes and the Hermitian phase operator expectation value is $2 \pi s /(2 s+1)$. 


\section{$3 \quad$ Unitary depolarizer operators and generalized Bell states}

As mentioned in the introduction the extended Weyl-Heisenberg algebra not only introduces mathematical tools for the analysis of finite quantum systems but also can be applied, for instance, to the context in quantum information processing via the so-called the unitary depolarizer operators and the generalized Bell states. In this section, we shall present the derivation of unitary depolarizer by means of phase operator. We also obtain generalized Bell states via the action of unitary phase operator and establish a relation between them and vectors generating mutually unbiased bases (MUB).

\subsection{Unitary depolarizers operators}

In a $d$-dimensional Hilbert space, the unitary depolarizers on a domain $\mathcal{D}$ are the elements of set

$$
S_{d}=\left\{U_{\alpha} \mid U_{\alpha} U_{\alpha}^{\dagger}=U_{\alpha}^{\dagger} U_{\alpha}=\mathbf{I}, \alpha \in \mathcal{D}\right\},
$$

which satisfy the relation

$$
\frac{1}{d} \sum_{\alpha \in \mathcal{D}} U_{\alpha} O U_{\alpha}^{\dagger}=(\operatorname{Tr} O) \mathbf{I}
$$

for any operator $O$ defined on the Hilbert space. The phase operator associated with extended WeylHeisenberg algebras discussed in the previous section offers a simple way to define unitary depolarizers. For this, we rewrite the unitary phase operator $E$ as

$$
E=\sum_{n=0}^{2 s} e^{i(F(n+1)-F(n)) \varphi}|n\rangle\langle n+1|,
$$

from which one can obtain

$$
E^{k}=\sum_{n=0}^{2 s} e^{i(F(n+k)-F(n)) \varphi}|n\rangle\langle n+k| \quad \text { modulo }(2 s+1) .
$$

The phase states (23) are eigenstates of the operator $E^{k}$. Indeed, one has

$$
E^{k}|m, \varphi\rangle=e^{i k \theta_{m}}|m, \varphi\rangle
$$

Any operator $O$, acting on the Fock space $\mathcal{F}$, can be expanded in the phase states basis $\{|m, \varphi\rangle, m \in$ $\mathbb{Z} /(2 s+1) \mathbb{Z}\}$ as follows

$$
O=\sum_{m=0}^{2 s} \sum_{m^{\prime}=0}^{2 s} O_{m, m^{\prime}}|m, \varphi\rangle\left\langle m^{\prime}, \varphi\right|
$$

It is simple to check that

$$
\sum_{k=0}^{2 s} E^{k} O E^{k^{\dagger}}=(2 s+1) \sum_{m=0}^{2 s} O_{m, m}|m, \varphi\rangle\langle m, \varphi| .
$$

Next, we define the unitary operator

$$
V(\varphi)=e^{i(F(N)+N) \varphi}
$$


in terms of the number operator and the structure function $F($.$) . Using the equations (49)-(50), the$ expression of the phase states (23) and performing integration over $\varphi$, one gets

$$
\frac{1}{2 \pi} \int_{-\pi}^{+\pi} d \varphi \sum_{k=0}^{2 s} V(\varphi) E^{k} O E^{k^{\dagger}} V(\varphi)^{\dagger}=\operatorname{Tr} O \mathbf{I}
$$

This constitutes a generalization of the definition of unitary depolarizers operators discussed in [15]. Then, the definition of unitary depolarizers (eqs. (43) and (44)) can be extended to unitary operators labeled by continuous parameters belonging to some compact domain. It is important to note that for quantized values of the parameter $\varphi$, given by

$$
\varphi=\frac{2 \pi}{2 s+1} l, \quad l \in \mathbb{Z} /(2 s+1) \mathbb{Z},
$$

one can verify that the discrete analogue of the equation (51) is

$$
\sum_{k=0}^{2 s} \sum_{l=0}^{2 s} V_{l} E^{k} O E^{k^{\dagger}} V_{l}^{\dagger}=(2 s+1) \operatorname{Tr} O \mathbf{I}
$$

where $V_{l} \equiv V(2 \pi l /(2 s+1))$. This agrees with the result obtained in [15]. So, the unitary depolarizer operators can be expressed in terms of the phase operator. To close this subsection, it is important to stress that the construction of unitary depolarizers is done without specifying the nature of the structure function $F($.$) . This provides us with a general scheme to define the unitary depolarizers$ for a large class of extended Weyl-Heisenberg algebras and can be adapted to quantum systems with higher symmetries.

\subsection{Generalized Bell states and MUB}

In this last part of our work, we shall focus on extended Weyl-Heisenberg algebra defined by the structure function

$$
F(N)=\frac{N}{2 s}(2 s+1-N) .
$$

In this case, we will show that the generalized Bell sates can be generated from the maximally entangled states using the phase operator. Recall that the generalized Bell states were first introduced in [16] to study quantum teleportation for higher dimensional quantum systems. We discuss briefly the entanglement properties of some particular superpositions of generalized Bell states and establish a connection between the generalized Bell states and the vectors of mutually unbiased bases.

The maximally entangled state of two $(2 s+1)$-dimensional systems is

$$
|\mathrm{MES}\rangle=\frac{1}{\sqrt{2 s+1}} \sum_{n=0}^{2 s}|n\rangle \otimes|n\rangle .
$$

The action of the operator $E^{k} \otimes \mathbf{I}$ on the state $|\mathrm{MES}\rangle$ leads to

$$
E^{k} \otimes \mathbf{I} \frac{1}{\sqrt{2 s+1}} \sum_{n=0}^{2 s}|n\rangle \otimes|n\rangle=\frac{1}{\sqrt{2 s+1}} \sum_{n=0}^{2 s} e^{i(F(n+k)-F(n)) \varphi}|n\rangle \otimes|n+k\rangle .
$$


Using (53) and setting $\varphi=-\frac{2 s}{2 s+1} \frac{p}{k} \pi$ (this discretization condition of $\varphi$ is similar to one introduced in [6] to get mutually unbiased states from phase states), one can write the equation (55) as

$$
E^{k} \otimes \mathbf{I} \frac{1}{\sqrt{2 s+1}} \sum_{n=0}^{2 s}|n\rangle \otimes|n\rangle=q^{\frac{p}{2}(k-2 s-1)}\left|\Psi_{k, p}\right\rangle,
$$

where the states $\left|\Psi_{k, p}\right\rangle$ are the generalized Bell states introduced in [16]. They are given by

$$
\left|\Psi_{k, p}\right\rangle=\frac{1}{\sqrt{2 s+1}} \sum_{n=0}^{2 s} q^{n p}|n\rangle \otimes|n+k\rangle .
$$

It follows that the generalized Bell states can be obtained from the maximally entangled states (54) by means of a successive application of the unitary phase operator.

Finally, it is interesting to note some characteristics of some particular superpositions of generalized Bell states $\left|\Psi_{k, p}\right\rangle$ and their expressions in terms of vectors belonging to mutually unbiased bases.

First, it is remarkable that the superposition of the $(2 s+1)^{2}$ maximally entangled Bell states

$$
\frac{1}{2 s+1} \sum_{k=0}^{2 s} \sum_{p=0}^{2 s}\left|\Psi_{k, p}\right\rangle=\frac{1}{\sqrt{2 s+1}}|0\rangle \otimes \sum_{n=0}^{2 s}|n\rangle .
$$

is a completely separable state. In other words, the superposed state is disentangled despite that it is a superposition of maximally entangled states. This is not always true. Indeed, the following superposition

$$
\sum_{k=0}^{2 s}\left|\Psi_{k, k}\right\rangle=\frac{1}{\sqrt{2 s+1}} \sum_{n=0}^{2 s}|n\rangle \otimes \sum_{k=0}^{2 s} q^{n k}|n+k\rangle .
$$

gives a maximally entangled state. The question concerning the entanglement of superposed Bell states is very rich and deserves more investigation. We hope to report on this issue in a forthcoming work. There is also a nice connection between the superposition of Bell states and the vectors of mutually unbiased bases $B_{p}$ introduced in [6] as

$$
B_{p}=\left\{\left|\phi_{m}^{p}\right\rangle=\frac{1}{\sqrt{2 s+1}} \sum_{n=0}^{2 s} q^{\frac{p}{2} n(2 s+1-n)} q^{n m}|n\rangle, m \in \mathbb{Z} /(2 s+1) \mathbb{Z}\right\} \quad p \in \mathbb{Z} /(2 s+1) \mathbb{Z} .
$$

Indeed, the superposed states (58) can be expressed in terms of the mutually unbiased vectors $\left|\phi_{m}^{p}\right\rangle$. This expression is

$$
\frac{1}{2 s+1} \sum_{k=0}^{2 s} \sum_{p=0}^{2 s}\left|\Psi_{k, p}\right\rangle=|0\rangle \otimes\left|\phi_{0}^{0}\right\rangle
$$

and the state (59) can be written as

$$
\sum_{k=0}^{2 s}\left|\Psi_{k, k}\right\rangle=\sum_{n=0}^{2 s} q^{-n^{2}}|n\rangle \otimes\left|\phi_{n}^{0}\right\rangle .
$$


The vectors $\left|\phi_{n}^{0}\right\rangle$ involved in the above superposition belong to the basis $B_{0}$. One can go further to find superpositions of maximally entangled states related to vectors $\left|\phi_{n}^{p}\right\rangle$ with $p \neq 0$. Indeed, it is easy to see that the action of the unitary operator $\mathbf{I} \otimes e^{i F(N) \delta}$, with $\delta=\frac{2 s}{2 s+1} p \pi$, on the states (62) gives

$$
\mathbf{I} \otimes q^{\frac{p}{2} N(2 s+1-N)}\left[\sum_{k=0}^{2 s}\left|\Psi_{k, k}\right\rangle\right]=\sum_{n=0}^{2 s} q^{-n^{2}}|n\rangle \otimes\left|\phi_{n}^{p}\right\rangle .
$$

This establishes a simple relation between the generalized Bell states and the vectors of the mutually unbiased bases. To close this section, we notice that the vectors of mutually unbiased bases $B_{p}$ can be defined as the quadratic Fourier transform of the Hilbert space basis [34]. Remark also that the generators of generalized Pauli group, which constitutes an important ingredient to deal with the relation between generalized Bell states and MUBs [24], can be defined as in 34].

\section{Concluding remarks}

This Letter is based on the extended Weyl-Heisenberg algebra. This algebra is interesting in many respects. It describes in a unified way a large class of generalized harmonic oscillators encountered in the literature. The extended Weyl-Heisenberg algebra, under some assumptions, possess finite dimensional representations. The nonlinear structure function characterizing the algebra can take into account some nonlinear effects that may occur in the quantum description of quantized modes of the electromagnetic field [35]. In connection with the extended Weyl-Heisenberg algebra, this work deals with the construction of a unitary phase operator and the determination of its temporally stable eigenstates (the so-called phase states). This construction is valid for a large class of generalized harmonic oscillators. We also discussed the derivation of unitary depolarizer operators from the unitary phase operators. We have shown that the formalism, presented in [15], using the Pegg-Barnett operator can be generalized to a large variety of extended harmonic oscillator. Finally, we discussed the generation of generalized Bell states [16] using the phase operator. We also investigated the degree of entanglement of superpositions of Bell states and established a connection between them and the vectors of mutually unbiased bases.

\section{Acknowledgments:}

M.D. would like to thank the hospitality and kindness extended to him by the Max Planck Institute for Physics of Complex Systems (Dresden, Germany) where this work was done.

\section{References}

[1] P.A.M. Dirac, Proc. R. Soc. London, Ser. A 114 (1927) 243.

[2] W.H. Louisell, Phys. Lett. 7 (1963) 60.

[3] L. Susskind and J. Glogower, Physics (U S) 49 (1964) 1.

[4] P. Carruthers and M.M. Nieto, Rev. Mod. Phys. 40 (1968) 411.

[5] D.T. Pegg and S.M. Barnett, Phys. Rev. A 39 (1989) 1665. 
[6] M. Daoud and M.R. Kibler, J. Phys. A: Math. Theor. 43 (2010) 115303.

[7] H-C. Fu, R. Sasaki, J. Phys. A: Math. Gen. 29 (1996)4049.

[8] C. Quesne and N. Vansteenkiste, Phys. Lett. A 240 (1998) 21; Int. J. Theor. Phys. 39 (2000) 1175.

[9] C. Quesne, Phys. Lett. A 272 (2000) 313; erratum Phys. Lett. A 275 (2000) 313; Mod. Phys. Lett. A 18 (2003) 515.

[10] T. Appl and D.H. Schiller, J. Phys. A: Math. Gen. 37 (2004) 2731.

[11] M. Daoud and M. Kibler, Phys. Lett. A 321 (2004) 147; J. Math. Phys. 47 (2006) 122108.

[12] T. Hiroshima, J. Phys. A: Math. Gen. 35 (2001) 6907.

[13] R.F. Werner, J. Phys. A: Math. Gen. 35 (2001) 7081.

[14] G. Bowen, Phys. Rev. A 63 (2001) 022302.

[15] M. Ban, J. Phys. A: Math. Gen. 35 (2002) L193.

[16] C.H. Bennett, G. Brassard, C, Crépeau, R. Jozsa, A. Peres and W. K. Wootters, Phys. Rev. Lett. 70 (1993) 1895.

[17] H. Bechmann-Pasquinucci and W. Tittel, Phys. Rev. A 61 (2000) 062308.

[18] M. Bourennane, A. Karlsson and G. Björk, Phys. Rev. A 64 (2001) 012306.

[19] N.J. Cerf, M. Bourennane, A. Karlsson and N. Gisin, Phys. Rev. Lett. 88 (2002) 127902.

[20] D. Sych, B. Grishanin and V. Zadkov, Phys. Rev. A 70 (2004) 052331.

[21] D. Sych and G. Leuchs, New J. Phys. 11 (2009) 013006.

[22] W.K. Wootters, Ann. Phys. (NY) 176 (1987) 1.

[23] W.K. Wootters and B.D. Fields, Ann. Phys. (NY) 191 (1989) 363.

[24] A.B. Klimov, D. Sych, L.L. Sanchez-Soto and G. Leuchs, Phys. Rev. A 79 (2009) 052101.

[25] A. Luis and L.L. Sanchez-Soto, Phys. Rev. A 48 (1993) 4702.

[26] A. Luis, Prog. Opt. 41 (2000) 421.

[27] P.W. Higgs, J. Phys. A: Math. Gen. 12 (1979) 309.

[28] E.K. Sklyanin, Funct. Anal. Appl. 16 (1982) 263.

[29] M. Rocek, Phys. Lett. B 255 (1991) 554.

[30] D. Bonatsos, C. Daskaloyannis and G. Lalazissi, Phys. Rev. A 47 (1993) 3448. 
[31] B. Abdesselam, J. Beckers, A. Chakrabarti and N. Debergh, J. Phys. A : Math. Gen. 29 (1996) 3075 .

[32] A.B. Klimov, L.L. Sanchez-Soto, A. Navarro and E.C. Yustas, J. Mod. Opt. 49 (2002) 2211.

[33] A. Vourdas, Phys. Rev. A 41 (1990) 1653.

[34] M.R. Kibler, J. Phys. A: Math. Theor. 42 (2009) 353001.

[35] D.F. Walls, Nature 306 (1983) 141. 